\title{
Protection of materials from hydrogen accumulation
}

\author{
Yuri A. Yakovlev ${ }^{1, *}$, Petr A. Zumberov ${ }^{1,2}$ \\ ${ }^{1}$ Institute for Problems in Mechanical Engineering of the Russian Academy of Sciences, 199178 V.O., Bolshoj pr., 61, St. Petersburg, \\ Russia \\ ${ }_{2}^{2}$ Peter the Great St. Petersburg Polytechnic University (SPbPU), 195251 Polytechnicheskaya, 29, St. Petersburg, Russia
}

\begin{abstract}
The article is concerned with possible accidents caused by high concentrations of hydrogen in metals. The problem of using recycled metal is considered. The mechanisms of hydrogen accumulation are described. Among other things, we propose an introduction to the output control of hydrogen concentrations. The results of reducing the concentration of diffuse-mobile hydrogen in steels after ultrasonic treatment are described.
\end{abstract}

\section{Introduction}

In the 19th century it was revealed that the dissolved hydrogen severely deteriorates properties of steels $\mathrm{cf}$. [1]. Later, it was figured out that the metallurgical hydrogen gets into steel while producing castings and is linked to the ingress of water and water-bearing infusions into stock in smelting cf. [2]. Despite such prolonged and thorough investigation of the hydrogen issue the problem still has not been solved-because of the steady developement of technology and metal fabrication. With every technological leap new problems of hydrogen and metal interaction are discovered. It is worth noting that in such case hydrogen concentration which has a significant influence on the mechanical properties of metal becomes lower. Thus, at the beginning of the examination of the hydrogen induced steel degradation it was considered that hydrogen embrittlement occurred when the concentration of hydrogen became more than $10-20 \mathrm{ppm}$. For some modern high strength steels the critical hydrogen content is established at the level of 0.1-0.2 ppm cf. [3].

The application of nano crystalline structures obtained from heatproof alloys used in gas-turbine engines or improvement of the engines operating performance serves a good example of the alteration of the hydrogen interaction character. Nanostructured single crystal does not have intercrystalline borders which are the sites for hydrogen accumulation cf. [4]. It eliminates the possibility of intercrystalline failure. However, it was found that in such oriented unit crystals other dangerous defects appear - micropores and microcracks induced by hydrogen which appear during crystallization process cf. $[5,6]$. This process is difficult to diagnose during production because of the cracks and voids have microscopic sizes. Nevertheless, they have a significant influence on the mechanical properties and on the operational reliability.
The modern technology development is correlated with lightweight constructions which are produced by involving the use of various ultrahigh strength or nanostructured materials. These materials have extreme mechanical characteristics. For example, the body of a modern automobile contains more than $50-70 \%$ of different kinds of high strength steels. Besides, this persentage will grow year by year, as high strength steels application in automobile manufacturing solves two problems at the same time: weight-saving and safety improvement. The application of high strength steels at the construction of gas pipe lines gives the opportunity to increase their capacity sinificantly. The experience of the application of high strength or nanostructured materials has revealed a very high degree of hydrogen impact on their mechanical properties cf. [7]. At the same time, the level of hydrogen concentration at which considerable influence on the mechanical properties begins has decreased by ten folds.

Thus, new approaches to diagnostics and the development of new protection against hydrogen accumulation methods are essential.

\section{Control of hydrogen concentration}

It should be noticed that there are two basic stages of critical hydrogen concentrations accumulation: during metal production and during exploitation.

During production hydrogen accumulates because of water-bearing admixtures, which get into the stock as well as right into the metal at processing of semimanufactured materials - water, oil, different additives, and so on.

The final hydrogen content checking at the castings should be carried out. Unfortunatelly, sometimes it is ignored, generally, for strain aluminum and titanium alloys.

\footnotetext{
* Corresponding author: yura.yakovlev@gmail.com
} 
Utilization of large amounts of recycled or secondary metal leads to the significant growth of hydrogen concentration during metal production in particular. Such growth of concentration may lead to the formation of hydrogen bubbles and fragility in alluminium alloys or to fragility and coldbrittleness of steels.

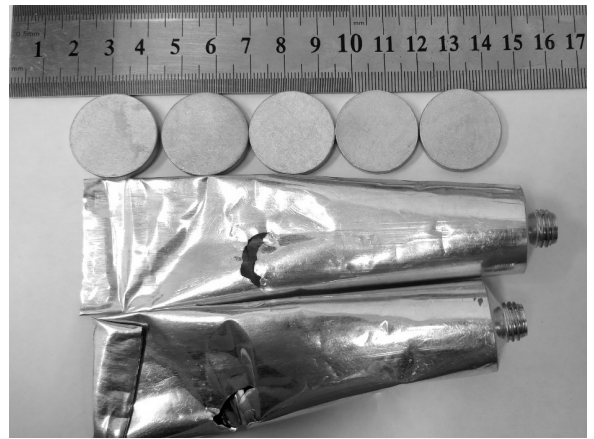

Fig. 1. The formation of hydrogen bubbles in alluminium.

The impossibility of performance of automatic and semiautomatic welding can be referred to other traits of hydrogen impact. High concentration of hydrogen in wire material leads to the weld cracking and sometimes even foaming. In aluminum alloys the seam foaming happens at relatively low hydrogen concentrations 0.5 $0.8 \mathrm{ppm}$ in the original material that being welded. But even if hydrogen concentrations in the original material fit the permitted values, accumulation is possible due to the air water dissociation and the hydrogen diffusion from the environment.

It is known that mechanical stresses lead to the diffusion of the alloy components into the tension stress area. We have investigated metal samples made of high strength steel after destruction. The hydrogen content in a bolt shank was 1-2 ppm. Such concentration is high but not critical for high strength steel. The bolts were covered with special anti-corrosion coat to avoid rust. The hydrogen concentration in the coat was about 1000 ppm. As a result, the diffusion of hydrogen from the coat into the bolt shank took place under the loading. This leads to the accumulation of the critical hydrogen concentration inside steel and its brittle failures. Consequently, the hydrogen control at different production stages is the only method of protection against such incidents.

For control and determination of hydrogen concentration we use the hydrogen analyzer AV-1. The precision hydrogen analyzer AV-1 has been designed for hydrogen detection in metals and alloys under the plant laboratory conditions during the outgoing control provided for different alloys [8-12]. The operating principle of the analyzer is mass-spectrometric.

The sample processing, as shown in figure 2, includes a vacuum extractor and a heater (4). The vacuum extractor is made of quartz glass and has three setoffs. The first setoff (1) contains the samples before the test. The hydrogen content analysis takes place in the second setoff (2). The samples which have been tested are kept in the third setoff (3).

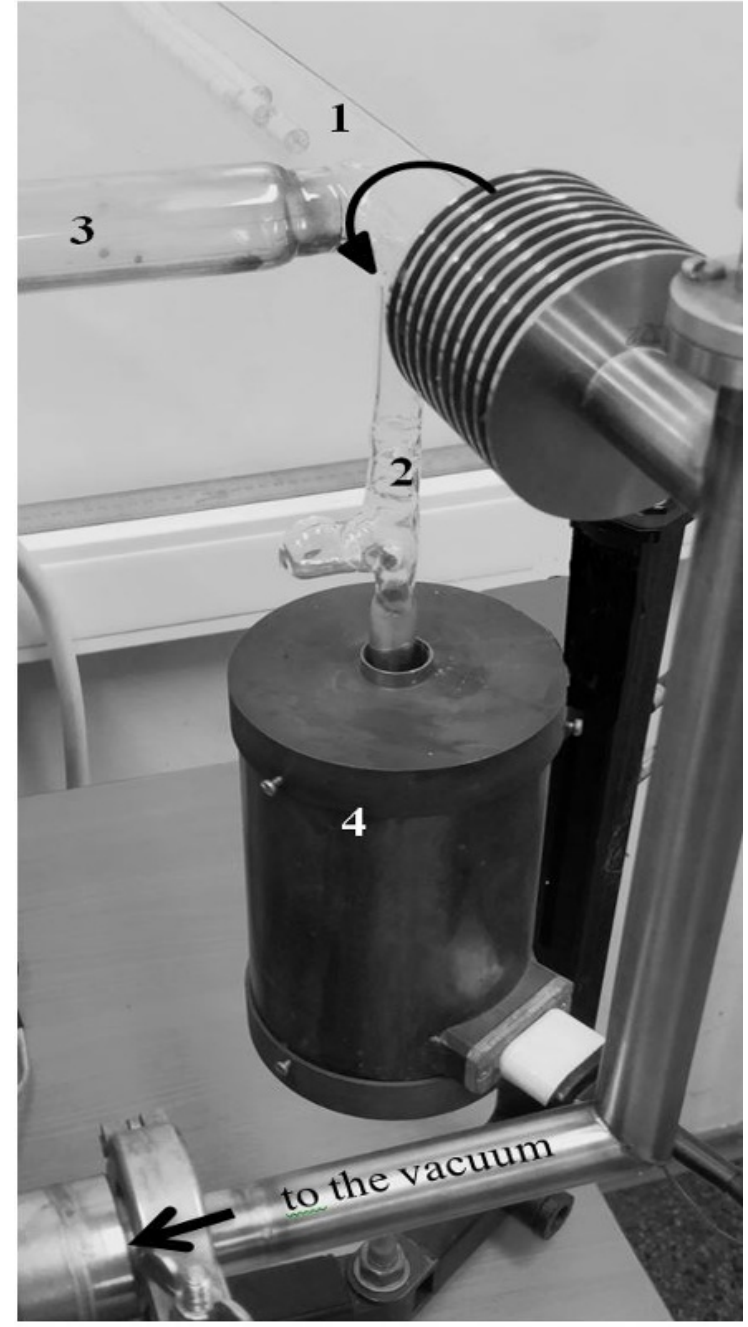

Fig. 2. The sample processing of hydrogen analyser AV-1.

Before testing, the heater (4) with previously set temperature is placed upon the second setoff (2) of the extractor. With the help of a magnetically push rod the test sample from the first setoff is thrown into the second analytic setoff of the extractor. During the test the metal sample within the second setoff is subjected to gradual heating up to the extraction temperature. The extraction temperature for the sample is lower than its melting temperature, as a rule, it is within the interval of 400$900^{\circ} \mathrm{C}$. The gases that have been emitted during the sample heating are evacuated from the extractor before the pressure reaches 50 micro $\mathrm{Pa}$ and are analyzed with a mass-spectrometer which is located in the vacuum pumping pass customized for the hydrogen line.

The end of the testing is identified by a background signal. The background signal remains constant before the beginning and at the end of the testing. As soon as the level of the signal from the sample becomes equal to the background signal - the test stops and the sample from the analytical setoff (2) is replaced into the setoff for the samples which have been analyzed (3). For this purpose, the heater (4) is lowered down by the hoisting gear, and the extractor keeps turning about itself until the sample being tested falls down from setoff (2) into setoff (3) under gravity. After that, the extractor is returned to the original position, and the heater is placed on setoff 
(2) with the help of hoisting gear. After setting the background hydrogen value the test continues for the next sample from setoff (1). In such manner, the next sample is analyzed without vacuum failure. It should be emphasized, that the extraction temperature does not change during the test. This makes it possible to reach high stability of the background signal.

The time dependence of hydrogen flow $q(\mathrm{t})$ is recorded by the system of digital registration as an extraction curve. Fig. 3 shows an extraction curve for aluminum alloy AMg-6, which is a simple example to describe.

Time integral from the extraction curve (painted grey in figure 3 ) in reference to background hydrogen flow is proportional to hydrogen evolved from the sample. The proportionality factor is set during analyzer calibration.

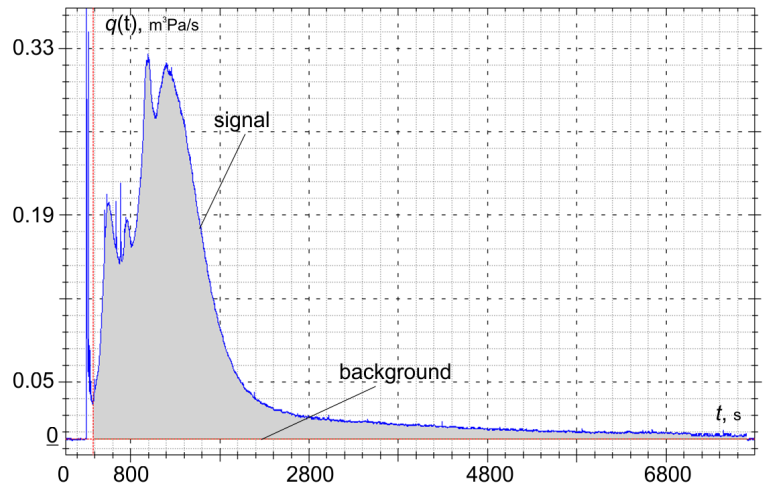

Fig. 3. Extraction curve for aluminum alloy AMg-6.

Each peak of the extraction curve can be correlated with its hydrogen binding energy [10].

\section{Decreasing the concentration of hydrogen during operation process}

During operation, due to various physical and chemical processes, the concentration of hydrogen increases. Accumulation of hydrogen in components of oil and gas pipelines is a potential threat, since it can lead to a brittle failure with a release of large amount of energy. This, in turn, can lead to human casualties and harm the environment. Unfortunately, nondestructive methods of hydrogen control do not exist, but there are methods that allow to reduce the hydrogen concentration by several times. One of these methods is Ultrasonic Peening treatment. Ultrasonic Peening (UP) is a promising technology in the application of High Power Ultrasound treatment for fatigue life improvement of welded structures $[12,13]$. The treatment is executed with a pin, from 2 to $5 \mathrm{~mm}$ in diameter made from high strength steel. The frequency of the oscillation of the pin is 20-30 $\mathrm{kHz}$, the total power is $300-500 \mathrm{~W}$, the amplitude of the oscillation is $40 \mu \mathrm{m}$. Ultrasonic Peening is usually applied to the adjunction of a weld and a heat-affected zone, as shown in Fig. 4.

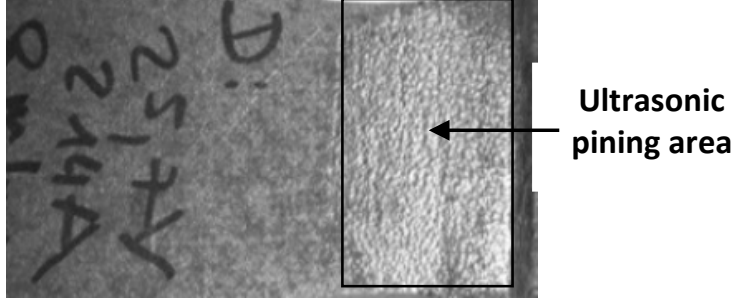

Fig. 4. Ultrasonic peening.

For the majority of steels, the concentration of diffusely mobile hydrogen is critically important. This hydrogen plays a key role in the formation and growth of cracks. In [14] the critical concentrations of diffusive hydrogen for crack growth are stated.

Our results of hydrogen diagnostics show, that one of the important results of UP treatment is almost triple reduction of low binding energy diffusive hydrogen.

Mechanical tests show, that in the samples, treated with UP, fatique cracks are not observed in the treared areas.

Thus, after ultrasonic peening, a significant reduction of diffusely mobile hydrogen occurs. In the studied samples, treated with UP, fatique cracks in the weld zone are not observed.

But diffusive hydrogen accumulates in the metal again during the process of cyclic loading. This effect is well known cf. [15]. Repeated treatment decreases the concentration by several times, which allows to prolong the service life of a construction. The study [15] shows, that in cyclic loading, the concentration of diffusely mobile hydrogen is of crucial importance. If the safe level is exceeded, development of local hydrogen fragility can occur by the mechanism of parametric resonance. Occasional treatment of the construction's components allows to decrease the concentration of diffusely mobile hydrogen and to keep it below the critical level, which positively affects the load capacity of a construction. Below, in Fig. 5, a dependency of hydrogen, extracted from a sample during the step heating procces in a vacuum (DTDS - descrete thermodisorption spectre cf. [9]) on temperature for Nickel Alloy 718 after 6 hours of synthetic aging at $780{ }^{\circ} \mathrm{C}$ is shown.

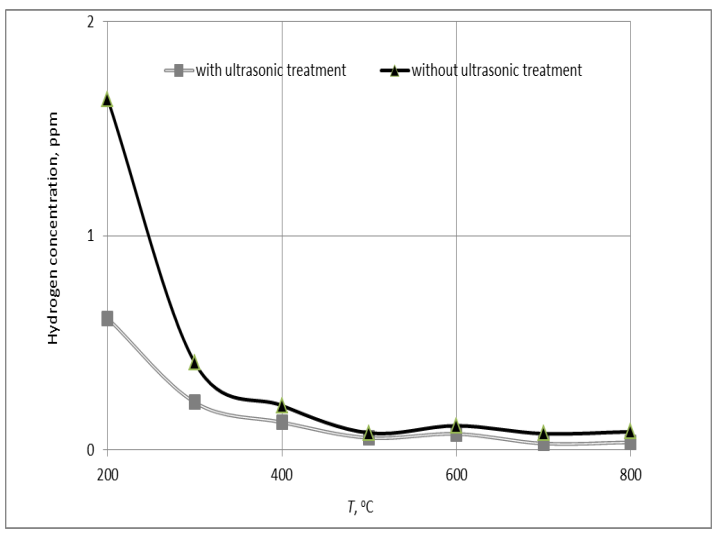

Fig. 5. DTDS of hydrogen concentration for nikel alloy 718 
The top curve represents a sample which was not ultrasonically treated, the bottom curve represents a sample after the ultrasonic treatment. Diffusely mobile hydrogen - hydrogen with a low binding energy [10], is released during heating in a vacuum at temperatures of $200-400{ }^{\circ} \mathrm{C}$. Comparing the two graphs, it is noticeable that the difference between the concentrations of diffusely mobile hydrogen in these two samples is twice greater.

\section{Conclusions}

According to the known facts about the impact of hydrogen on structure and properties of materials, it can be concluded that fatigue failure and accumulation of hydrogen are tightly connected.

As the tests show, a significant change in mechanical properties of materials occurs when the natural concentration of hydrogen is twice exceeded. This accumulation can occur in short time. Meanwhile, the material fails fragilely, without a formation of a main cracks.

The use of high proportions of recycled metals in metallurgic industry leads to a significant increase in the concentration of hydrogen. This increase leads to defects in components manufactured with previously proven technologies.

Measuring the concentration of hydrogen during production will allow to create a system of strict control, which will help to decrease variations in characteristics and reject parts and fasteners with high hydrogen content, low mechanical and corrosion properties.

\section{Acknowledgement}

The study has been undertaken with support of Russian Foundation for Basic Research grants: 18-3100329, 18-08-00201, 17-08-00783.

\section{References}

1. Fremy M. E. On the composition of cast iron and steel Journal of the Franklin Institute. - 1861. Vol. 72. - No. 5. - P. 342-346.

2. Andrew J. H. The embrittling of iron by caustic soda Transactions of the Faraday Society. - 1914. - Vol. 9. - P. 316-329.

3. Motomichi Koyama, et. al., Overview of hydrogen embrittlement in high-Mn steels International Journal of Hydrogen Energy.-2017.- Vol. 42, No. 17. - P. 12706-12723

4. Balyts'kyi A. I. et al. Corrosion-and HydrogenResistance of Heat-Resistant Blade Nickel-Cobalt Alloys Materials Science. - 2018. - Vol. 54, No 2. P. 230-239.

5. Ramirez A. J., Lippold J. C. High temperature behavior of Ni-base weld metal: Part II-Insight into the mechanism for ductility dip cracking Materials Science and Engineering: A. - 2004. - Vol. 380, No.
1-2. - P. 245-258.

6. Zhao L. G., O’Dowd N. P., Busso E. P. A coupled kinetic-constitutive approach to the study of high temperature crack initiation in single crystal nickelbase superalloys Journal of the Mechanics and Physics of Solids. - 2006. - Vol. 54, No 2. - P. 288309.

7. Koyama $M$. et al. Overview of hydrogen embrittlement in high-Mn steels International journal of Hydrogen Energy. - 2017. - Vol. 42, No17. - P. 12706-12723

8. Konar J., Banerjee N. G. Vacuum heating hydrogen determination in aluminum and aluminum alloys // NML Technical Journal. - 1974. - Vol. 16, No. 1-2. - P. 18-19

9. A. K. Belyaev, et al. Multichannel diffusion vs TDS model on example of energy spectra of bound hydrogen in 34CrNiMo6 steel after a typical heat treatment International Journal of Hydrogen Energy. - 2016. - Vol. 41, No. 20. - P. 8627-8634.

10. Polyanskiy A. M., Polyanskiy V. A., Yakovlev Yu. A. Experimental determination of parameters of multichannel hydrogen diffusion in solid probe International Journal of Hydrogen Energy. - 2014. - Vol. 39, No. 30. - P. 17381-17390.

11. D.Yu. Andronov, D.G. et al. Application of multichannel diffusion model to analysis of hydrogen measurements in solid International Journal of Hydrogen Energy. - 2017. - Vol. 42, No. 1. - P. 699-710

12. Y.Kudryavtsev, J. Kleiman, Fatigue improvement of welded elements and structures by ultrasonic peening ASME 2013 Pressure Vessels and Piping Conference. - American Society of Mechanical Engineers, 2013. - p.V06AT06A060

13. Zhang $H$. et al. Effects of ultrasonic impact treatment on pre-fatigue loaded high-strength steel welded joints International Journal of Fatigue. 2015. - Vol. 80 . - P. 278-287.

14. Shvachko V. I. Cold cracking of structural steel weldments as reversible hydrogen embrittlement effect International journal of hydrogen energy. 2000. - Vol. 25, No. 5. - P. 473-480.

15. A.K. Belyaev, et al. Parametric instability in cycling loading as the cause of fracture of hydrogenous materials. Mechanics of Solids. -T. 47.-. 5. - P. 533537 (2012) 